\title{
Type 2 diabetes mellitus - genes or intrauterine environment? An embryo transfer paradigm in rats
}

\author{
R. Gill-Randall ${ }^{1}$ D. Adams ${ }^{1}$ - R. L. Ollerton ${ }^{2}$ M. Lewis ${ }^{1}$ J. C. Alcolado ${ }^{1}$ \\ ${ }^{1}$ Department of Medicine, University of Wales College of Medicine, Cardiff, Wales, United Kingdom \\ 2 School of Quantitative Methods and Mathematical Sciences, University of Western Sydney, Penrith Campus, New South Wales, \\ Australia
}

\begin{abstract}
Aims/hypothesis. The familial predisposition to Type 2 diabetes mellitus is mediated by both genetic and intrauterine environmental factors. In the normal course of events, maternal genes always develop in the same uterus, thus restricting studies aimed at investigating the relative contribution of these factors. We have developed an embryo transfer paradigm in rats to overcome this difficulty.

Methods. Euglycaemic female Wistar rats were superovulated and mated with male Wistar rats. The following day, fertilised eggs were transferred into pseudopregnant female Wistar rats or hyperglycaemic Goto Kakizaki (GK) rats. Pregnancies were allowed to go to term. Offspring were weighed at 6 weeks, 3 months and 6 months of age and an intravenous glucose tolerance test was carried out at 6 months of age.

Results. Offspring from Wistar into Wistar embryo transfers $(n=20)$ were not significantly hyperglycaemic compared to the non-manipulated Wistar stock colony $(n=26)$. However, offspring from Wistar gametes
\end{abstract}

reared in hyperglycaemic GK mothers $(n=51)$ were significantly lighter at 6 weeks of age $(156 \pm 4.1 \mathrm{~g}$ vs $180 \pm 6.1 \mathrm{~g}$ [mean $\pm \mathrm{SEM}], p<0.01)$ and significantly more hyperglycaemic at 6 months of age (fasting glucose $6.6 \pm 0.18 \mathrm{mmol} / 1$ vs $4.8 \pm 0.21 \mathrm{mmol} / 1$, mean

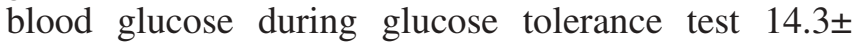
$0.31 \mathrm{mmol} / 1$ vs $11.1 \pm 0.28 \mathrm{mmol} / \mathrm{l}, p<0.01$ ) than Wistar gametes transferred back into euglycaemic Wistar mothers. When GK rats were superovulated and mated together, transfer of 1-day-old embryos into pseudopregnant Wistar dams did not alleviate hyperglycaemia in adult offspring.

Conclusions/interpretation. In GK rats, a euglycaemic intrauterine environment cannot overcome the strong genetic predisposition to diabetes. However, in Wistar rats with a low genetic risk of diabetes, exposure to hyperglycaemia in utero significantly increases the risk of diabetes in adult life.

Keywords Embryo transfer - Genetics - Intrauterine environment $\cdot$ Superovulation
Received: 28 January 2004 / Accepted: 14 May 2004

Published online: 17 July 2004

(C) Springer-Verlag 2004

J. C. Alcolado (৫)

Department of Medicine,

University of Wales College of Medicine,

Heath Park, Cardiff, Wales, CF14 4XN, United Kingdom

E-mail: Alcolado@btinternet.com

Tel.: +44-29-20747247

Abbreviations: AvGTT, weighted average blood glucose during glucose tolerance test · FG, fasting blood glucose . GK, Goto Kakizaki · W, Wistar

\section{Introduction}

Type 2 diabetes mellitus is a familial disorder and numerous studies have reported that patients more frequently recall that they had an affected mother than an affected father [1,2]. Several mechanisms could explain this pattern of inheritance, including the influences of maternally inherited genes and of the intrauterine environment.

Evidence for a genetic cause includes the discovery of a subgroup of diabetes associated with maternally transmitted mitochondrial DNA defects $[3,4,5]$. The role of the intrauterine environment is complex. The 
"thrifty phenotype" hypothesis suggests that impaired fetal nutrition may increase susceptibility to diabetes in later life $[6,7]$. In contrast, Freinkel coined the term "fuel-mediated teratogenesis" for the process by which adverse effects arise in the offspring of diabetic mothers as a consequence of excessive exposure to glucose within the uterus [8], including an increased susceptibility to diabetes in later life. Thus offspring of Pima Indians who were in-utero when their mother had diabetes have a greater risk of diabetes than older siblings born before the mother developed diabetes [9].

Since maternal genes normally always develop in the same uterus, research studies into the relative contributions of genes and intrauterine environment have proved difficult to design. A few studies have attempted to address this important issue directly. Dietary manipulations, such as protein restriction, of pregnant rodents have been undertaken in an attempt to alter the intrauterine environment whilst keeping a constant genetic background $[10,11]$. Offspring of rats rendered diabetic by the administration of streptozotocin are more likely to develop diabetes than pups from litters born prior to the manipulation [12].

However, these experimental approaches have limitations. For example, it has not been possible to fully study the effects of rearing offspring at genetically high risk of diabetes in a normal uterus, as it is difficult to maintain perfect euglycaemia in diabetic rats (except perhaps transiently with islet cell transplantation [13]). The confounding effects of maternal age and parity must also be taken into account.

The aim of our studies was to establish an embryo transfer system to examine the relative contribution of genetic factors and of the intrauterine environment to the later development of diabetes.

Given the nature of our proposed experiments, we chose a rodent model with Type 2 diabetes, the Goto Kakizaki (GK) rat. This strain was developed by the successive inbreeding of Wistar rats with the highest blood glucose concentrations [14]. Adult GK rats develop stable moderate hyperglycaemia. We chose this strain for several reasons. Firstly, the ancestral Wistar strain is readily available and hence we did not foresee a species block or compatibility problem with embryo transfer. Secondly, adult GK rats have a relatively normal lifespan with no requirement for specific treatment of their hyperglycaemia. Thirdly, the strain has been extensively studied before, including two genome-wide scans that have located potential genetic loci contributing to disease susceptibility [15, 16]. However, the GK rat does not provide a perfect model of human Type 2 diabetes. In particular, it is not significantly overweight.

\section{Materials and methods}

Experimental procedures. All procedures were carried out in accordance with licences granted under the British Animal (Surgical Procedures) Act. Outbred Wistar and GK breeding stock were maintained in-house at the BioMedical Services Unit of the University of Wales, College of Medicine, a designated breeding and animal research establishment. They were given free access to standard rat chow. We adhered to standard husbandry procedures with a 12-h light/dark cycle. All animals were identified by the insertion of a subcutaneous microchip.

To induce superovulation, we administered 5 IU of pregnant mare's serum into the peritoneal cavity of 3-month-old virgin female rats, which were restrained, but not anaesthetised. This was followed, $48 \mathrm{~h}$ later, by the intraperitoneal administration of $5 \mathrm{IU}$ of human chorionic gonadotrophin. We then caged up to four of the female rats with a proven male of the same strain and allowed mating to occur overnight. The following morning, coitus was confirmed by the presence of a vaginal plug and we killed these female rats by cervical dislocation. We immediately opened the abdomen, identified the oviducts and retrieved the fertilised eggs under an operating microscope. We cleaned the eggs of surrounding cumulus cells by incubating with a dilute solution of hyaluronidase in M3 medium.

We prepared recipient female rats by rendering them pseudo-pregnant. This was achieved by allowing 3-month-old virgin female rats to mate overnight with vasectomised Wistar rats. We performed the vasectomies under isoflurane anaesthesia, and success was judged by the repeated inability of these male rats to fertilise multiple female partners. Plugged recipient female rats were anaesthetised with isoflurane delivered in oxygen by a veterinary anaesthetic machine and we then accessed the oviducts through sequential bilateral paralumbar incisions. We transferred 6 to 8 of the freshly collected embryos into each ampulla using a micropipette primed with M3 media. These rats were allowed to recover and pregnancies continued until spontaneous delivery at term.

We attempted morphometric assessment at birth, but this resulted in an unacceptably high maternal rejection rate. For similar reasons, we did not pursue cross-fostering studies and all offspring were fed by their respective (uterine) mothers until weaned. Offspring were weighed at 6 weeks, 3 months and 6 months of age. At 6 months of age, offspring underwent an intravenous glucose tolerance test. After an overnight fast, and under isoflurane anaesthesia, one of the external jugular veins was cannulated and a baseline blood sample was taken. We then administered $0.8 \mathrm{~g} / \mathrm{kg}$ of glucose by rapid injection. We took blood samples at 5, 10, 15, 20 and 30 min for glucose estimations via tail-snips. In accordance with our licences, rats were then killed and tissue stored for subsequent analysis. Glucose tolerance tests were also done on a random selection of 6-month-old Wistar and GK rats from our breeding colonies.

Experiment groups. Three groups of experiments were carried out. Firstly, embryos obtained from Wistar rats were transferred back into pseudo-pregnant Wistar female rats $(\mathrm{W} \rightarrow \mathrm{W})$. This was our control group, to confirm that superovulation and embryo transfer would not be diabetogenic. Secondly, embryos obtained from Wistar rats were transferred into pseudo-pregnant $\mathrm{GK}$ females $(\mathrm{W} \rightarrow \mathrm{GK})$. The aim of this group was to study the effects of rearing embryos at low genetic risk of diabetes in a diabetic uterus. Finally, embryos from GK rats were transferred into Wistar uterus to study the effects of rearing embryos at high genetic risk of diabetes in a euglycaemic uterus $(\mathrm{GK} \rightarrow \mathrm{W})$. 


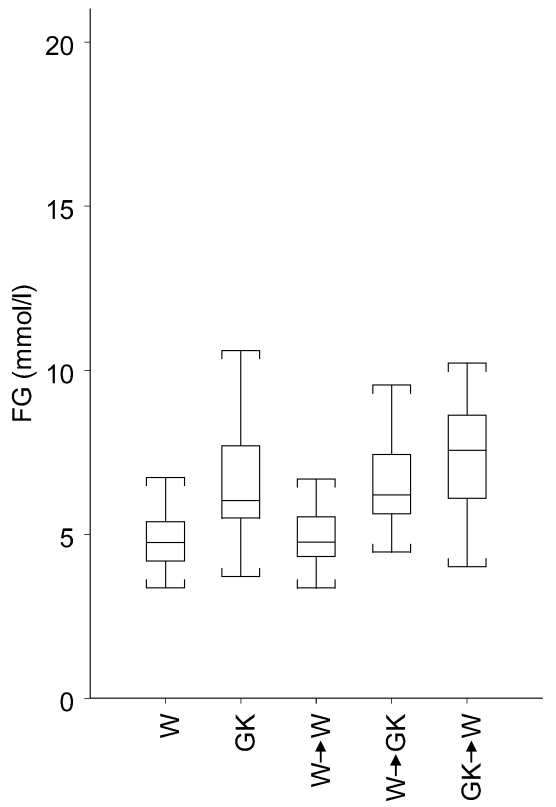

Fig. 1. Fasting blood glucose (FG) and average blood glucose during glucose tolerance test at 6 months of age (AvGTT) for Wistar stock colony, GK stock colony, and embryo transfer experiments. The central line of the box-plot indicates the median, the box indicates the interquartile range and the "whiskers" indicate the total range. Outliers are not shown. The AvGTT comparisons were unchanged when corrected for FG. Precise means \pm SEMs are given in text. W, Wistar stock colony; GK, Goto Kakizaki stock colony; W $\rightarrow$ W, Wistar embryos transferred into Wistar uterus; W $\rightarrow$ GK Wistar embryos transferred into GK uterus; GK $\rightarrow \mathrm{W}$, GK embryos transferred into Wistar uterus

Blood samples. All blood samples were coded and analysed by an external laboratory that was blinded to the source of the samples. Glucose was measured on a Vitros 250 automated analyser (Ortho Clinical Diagnostics, Johnson \& Johnson, Dublin, Ireland) that is calibrated and quality-controlled to UK clinical standards.

Statistical analysis. Data are presented as means \pm SEM. Normality assumptions were checked graphically. A $p$ value of less than 0.05 was taken to indicate statistical significance. Since the blood glucose value at each time point of an intravenous glucose tolerance test is not independent of the previous time point value and groups were significantly different in terms of fasting blood glucose, glucose tolerance data were summarised by using the weighted average glucose value between $t=0$ and $t=30 \mathrm{~min}$ (AvGTT, area under the curve calculated by the trapezoidal rule divided by 30 ). As each individual offspring was part of a litter and data from different offspring of the same litter could not be considered as independent, data were analysed by ANOVA, with the litter variable nested in the rat type variable.

\section{Results}

Wistar and Goto Kakizaki stock colonies. As expected, the GK stock colony was more hyperglycaemic than the Wistar colony. The mean fasting blood

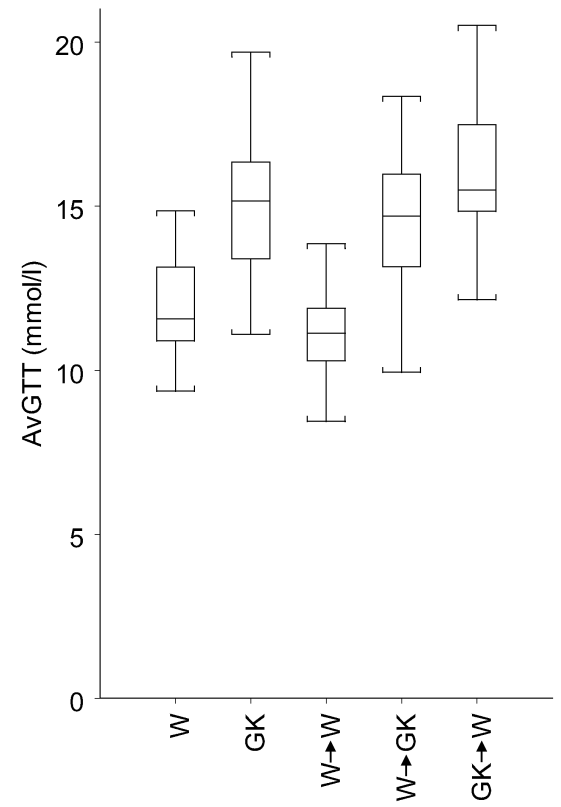

glucose (FG) concentration in Wistar rats was $4.9 \pm 0.22 \mathrm{mmol} / \mathrm{l}(n=26)$ compared to $6.9 \pm 0.45 \mathrm{mmol} / \mathrm{l}$ $(n=29)$ in GK rats (mean \pm SEM, $p<0.01)$. The mean AvGTT during the glucose tolerance test was also greater in the GK colony than in the Wistar colony $(14.9 \pm 0.47 \mathrm{mmol} / \mathrm{l}$ vs $12.0 \pm 0.28 \mathrm{mmol} / 1, p<0.01)$ (Fig. 1). These differences remained significant when data were analysed separately for female and male rats.

Wistar embryos transferred into Wistar uterus (Group 1). To assess any possible effect of superovulation and embryo transfer on glucose tolerance in adults, we studied 20 (9 male) offspring from two litters of Wistar into Wistar embryo transfers $(\mathrm{W} \rightarrow \mathrm{W})$ and compared FG and AvGTT during the intravenous glucose tolerance test at 6 months of age with FG and AvGTT values in our stock Wistar colony (Fig. 1). FG was similar in both groups (Wistar stock $4.9 \pm 0.22 \mathrm{mmol} / \mathrm{l}$ vs $\mathrm{W} \rightarrow \mathrm{W} 4.8 \pm 0.21 \mathrm{mmol} / \mathrm{l}$ ) for all animals and also when data were analysed separately for male and female rats. There was a small (but statistically significant at $p<0.05)$ reduction in AvGTT for $\mathrm{W} \rightarrow \mathrm{W}$ offspring compared to the Wistar stock colony (Wistar stock $12.0 \pm 0.28 \mathrm{mmol} / \mathrm{l}$ vs $\mathrm{W} \rightarrow \mathrm{W}$ $11.1 \pm 0.28 \mathrm{mmol} / \mathrm{l})$, but this did not reach significance when male or female rats were analysed separately. Thus, overall there was no evidence that the experimental procedures themselves would predispose to diabetes in offspring.

Goto Kakizaki embryos transferred into Wistar uterus (Group 2). We studied 41 (25 male) offspring from eight litters. We confirmed the euglycaemic status of the Wistar dams by performing an intravenous glucose tolerance test post-partum. The weight of offspring at 6 weeks, 3 months and 6 months of age is given in Table 1. At 6 weeks of age, $\mathrm{GK} \rightarrow \mathrm{W}$ embryo transfer offspring were significantly lighter than $\mathrm{W} \rightarrow \mathrm{W}$ em- 
Table 1. Mean weight (g) of embryo transfer offspring

\begin{tabular}{lll}
\hline & Male rats $(n)$ & Female rats $(n)$ \\
\hline Six weeks & & \\
$\mathrm{W} \rightarrow \mathrm{W}$ & $202 \pm 4.0(9)$ & $160 \pm 6.1(10)$ \\
$\mathrm{GK} \rightarrow \mathrm{W}$ & $164 \pm 8.8(25)^{\mathrm{a}}$ & $126 \pm 4.3(16)^{\mathrm{a}}$ \\
$\mathrm{W} \rightarrow \mathrm{GK}$ & $172 \pm 5.4(26)^{\mathrm{a}}$ & $139 \pm 4.2(25)^{\mathrm{a}}$ \\
$\mathrm{Three} \mathrm{months}$ & & \\
$\mathrm{W} \rightarrow \mathrm{W}$ & $333 \pm 8.4(9)$ & $236 \pm 12.4(11)$ \\
$\mathrm{GK} \rightarrow \mathrm{W}$ & $330 \pm 5.5(25)$ & $231 \pm 6.6(16)$ \\
$\mathrm{W} \rightarrow \mathrm{GK}$ & $400 \pm 9.0(26) \mathrm{b}$ & $238 \pm 4.1(25)$ \\
$\mathrm{Six} \mathrm{months}$ & & \\
$\mathrm{W} \rightarrow \mathrm{W}$ & & $291 \pm 21.1(11)$ \\
$\mathrm{GK} \rightarrow \mathrm{W}$ & $437 \pm 12.8(9)$ & $278 \pm 12.0(16)$ \\
$\mathrm{W} \rightarrow \mathrm{GK}$ & $408 \pm 6.0(25)$ & $275 \pm 4.8(25)$ \\
\hline
\end{tabular}

Data shown as means \pm SEM. a $p<0.001$ when compared to $\mathrm{W} \rightarrow \mathrm{W} ;{ }^{\mathrm{b}} p<0.05$ when compared to $\mathrm{W} \rightarrow \mathrm{W}$ and $\mathrm{GK} \rightarrow \mathrm{W}$; ${ }^{\mathrm{c}} p<0.05$ when compared to $\mathrm{GK} \rightarrow \mathrm{W}$

bryo transfer offspring but this was no longer apparent at 3 months of age.

At 6 months of age, FG and AvGTT during the intravenous glucose tolerance test were significantly higher in $\mathrm{GK} \rightarrow \mathrm{W}$ embryo transfer offspring (FG 8.0 $\pm 0.59 \mathrm{mmol} / \mathrm{l}$, AvGTT $16.1 \pm 0.30 \mathrm{mmol} / \mathrm{l}$ ) than in the Wistar stock colony (FG $4.9 \pm 0.22 \mathrm{mmol} / \mathrm{l}$, AvGTT $12.0 \pm 0.28 \mathrm{mmol} / \mathrm{l})$ or in the $\mathrm{W} \rightarrow \mathrm{W}$ embryo transfer offspring (FG 4.8 $\pm 0.21 \mathrm{mmol} / \mathrm{l}$, AvGTT $11.1 \pm 0.28 \mathrm{mmol} / \mathrm{l})(p<0.01$, Fig. 1$)$.

Wistar Rats transferred into Goto Kakizaki uterus (Group 3). We studied 51 (26 male) offspring from ten litters. We confirmed the diabetic status of the GK dams by performing an intravenous glucose tolerance test post-partum. The weight of offspring at 6 weeks, 3 months and 6 months of age is given in Table 1. At 6 weeks of age, GK $\rightarrow$ W embryo transfer offspring were of similar weight to $\mathrm{W} \rightarrow \mathrm{GK}$ offspring, but significantly lighter than $\mathrm{W} \rightarrow \mathrm{W}$ offspring. However, by 3 months of age, male $\mathrm{GK} \rightarrow \mathrm{W}$ offspring were heavier than male rats in both of these other groups. Although this difference appeared to persist at 6 months of age, statistical significance at $p<0.05$ was not maintained.

At 6 months of age, FG and AvGTT during the intravenous glucose tolerance test were significantly higher in the offspring of $\mathrm{W} \rightarrow \mathrm{GK}$ embryo transfers (FG $6.6 \pm 0.18 \mathrm{mmol} / \mathrm{l}$, AvGTT $14.3 \pm 0.31 \mathrm{mmol} / \mathrm{l}$ ) than in those of the Wistar rats in our stock colony and in the $\mathrm{W} \rightarrow \mathrm{W}$ embryo transfer control group (Fig. 1, $p<0.01$ ).

\section{Discussion}

We performed a series of superovulation and embryo transfer experiments in Wistar and GK rats. Our results confirm the importance of genetic factors in the development of Type 2 diabetes, independent of the intrauterine environment, since GK offspring were more hyperglycaemic than Wistar animals, regardless of whether or not they had been reared in a diabetic uterus.

GK embryos reared in a euglycaemic Wistar uterus are still destined to develop diabetes, suggesting that a strong genetic predisposition to diabetes cannot be alleviated even if intrauterine euglycaemia is achieved. It has been proposed that one benefit of tight glycaemic control during gestational diabetes in humans is a possible reduction in the risk of diabetes to offspring. Our results suggest that this benefit may be limited in families with a very high genetic risk for diabetes.

Superovulation and embryo transfer in themselves do not seem to be diabetogenic since the blood glucose concentrations in the $\mathrm{W} \rightarrow \mathrm{W}$ offspring were no higher than in the stock Wistar colony. Although we studied fewer animals than in our other groups, the techniques are routinely used in the generation of transgenic animals throughout the world and hyperglycaemia has not been reported as a complication. However, the techniques represent major interventions in early life and, although our results are reassuring and act as a control for our other embryo transfer groups, it is important to note that the currently described experiments were not designed to fully explore the possible diabetogenic role of superovulation and embryo transfer techniques. Potential mechanisms could include effects on ovum maturation and release, embryo manipulation, abnormal implantation or placentation. The litter sizes in our $\mathrm{W} \rightarrow \mathrm{GK}$ and $\mathrm{GK} \rightarrow \mathrm{W}$ groups were both similar but significantly smaller than that of the $\mathrm{W} \rightarrow \mathrm{W}$ group. This suggests that the technique is most successful when both genetic parents and pseudo-pregnant recipient female rats do not have diabetes and further studies are warranted to examine the mechanisms that underlie this observation.

Adult offspring of Wistar rat embryos transferred into a GK uterus were significantly more hyperglycaemic than the non-manipulated Wistar rats in our breeding colony and also the $\mathrm{W} \rightarrow \mathrm{W}$ embryo transfer control group. This suggests that, although genetic factors are pre-eminent in mediating diabetes in the offspring of GK rats, intrauterine environment plays an important role in predisposing to diabetes in rats at genetically low risk of diabetes. These results are in keeping with the higher prevalence of diabetes observed in the offspring of rats rendered diabetic by streptozotocin treatment [12] and data from adult children of Pima Indians with gestational diabetes [9].

Several studies have crossed non-diabetic male rats with diabetic GK rats, for example, as part of genome-wide searches, and the resulting offspring are invariably more hyperglycaemic than the original non-diabetic male colony $[15,16]$. One study has reported that offspring of Wistar male/GK female 
crosses are more hyperglycaemic than those of Wistar female/GK male crosses and this has also been taken as evidence of the diabetogenic effects of being exposed to diabetes in-utero [17]. However, these maternal effects have not been confirmed in other studies $[18,19]$ and the experiments are hampered by the fact that the offspring also share the maternal (non-diabetogenic) genes. Our embryo-transfer model removes this potential confounding factor, since offspring have not inherited genetic material from their surrogate mothers.

We postulate that hyperglycaemia in adult offspring of $\mathrm{GK} \rightarrow \mathrm{W}$ embryo transfers is primarily genetically mediated and that of $\mathrm{W} \rightarrow \mathrm{GK}$ transfers is modulated by the intrauterine environment. Our data give further support to the concept of fuel-mediated teratogenesis, in which it is postulated that intrauterine exposure to hyperglycaemia (or related factors such as excessive NEFA levels) causes long-term damage to the developing embryo. Several mechanisms might underlie this phenomenon. Intrauterine hyperglycaemia might mediate the permanent switching on or off of genes in the developing embryo, perhaps resulting in a survival advantage in utero but at the expense of increasing the risk of diabetes in adult life. Pancreatic beta cell mass or regenerative capacity might also be altered, increasing the risk of diabetes in later life. Imprinting, the preferential activation of genes inherited from either the mother or father, could be influenced by the prevailing intrauterine environment. Several genetic loci thought to be important in diabetes susceptibility are known to be imprinted [20]. Embryo transfer techniques are particularly well suited to the study of imprinting since both maternal and paternal genes can be experimentally controlled independent of the intrauterine environment.

In humans, early neonatal ingestion of breast milk from diabetic mothers may increase the risk of becoming overweight and consequently developing impaired glucose tolerance during childhood [21]. We were not able to perform cross-fostering experiments in rats due to high maternal rejection rates, especially amongst the GK mothers. Further studies, perhaps using expressed milk and assisted feeding may help quantify the relative contributions of intrauterine environment, early post-natal feeding and milk composition to the increased risk of diabetes in later life.

Although we were unable to carry out anthropometric measurements on our newborn pups, we made an intriguing observation that both $\mathrm{W} \rightarrow \mathrm{GK}$ and $\mathrm{GK} \rightarrow \mathrm{W}$ embryo transfer offspring were lighter at 6 weeks of age than the $\mathrm{W} \rightarrow \mathrm{W}$ control group. It is pertinent to note that human babies born as a result of superovulation and embryo transfer procedures are also lighter than control populations [22]. The study of human families born as a result of assisted reproduction technology may provide valuable insights into the relative contribution of genes and the intrauterine environment in diabetes, but until ethical and confidentiality issues are overcome [23], rodent embryo transfer studies such as ours will help disentangle the continuing debate regarding the influences of nature and nurture in metabolic disorders.

Acknowledgements. This work was funded by a Project Grant from Diabetes UK. We thank Dr D. Hullen and Dr S. Davies and the staff of the Biochemistry laboratory at the Royal Glamorgan Hospital for the blood glucose analyses.

\section{References}

1. Alcolado JC, Laji K, Gill-Randall R (2002) Maternal transmission of diabetes. Diabet Med 9:89-98

2. Alcolado JC, Alcolado R (1991) Importance of maternal history of non-insulin dependent diabetic patients. BMJ 302:1178-1180

3. Alcolado JC, Majid A, Brockington M et al. (1994) Mitochondrial gene defects in patients with NIDDM. Diabetologia 37:372-376

4. Van den Ouweland JM, Lemkes HH, Trembach RC et al. (1994) Maternally inherited diabetes and deafness is a distinct subtype of diabetes and associates with a single point mutation in the mitochondrial tRNA(leu(UUR)) gene. Diabetes 43:746-751

5. Suzuki Y, Suzuki S, Hinokio Y et al. (1997) Diabetes associated with a novel 3264 mitochondrial tRNA(leu)(UUR) mutation. Diabetes Care 20:1138-1140

6. Hales CN, Barker DJ, Clark PM et al. (1991) Fetal and infant growth and impaired glucose tolerance at age 64 . BMJ 303:1019-1022

7. Hales CN, Barker DJ (2001) The thrifty phenotype hypothesis. Br Med Bull 60:5-20

8. Metzyer BE (1991) Biphasic effects of maternal metabolism on fetal growth. Quintessential expression of fuelmediated teratogenesis. Diabetes 40 [Suppl 2]:99-105

9. Dabelea D, Knowler WC, Pettitt DJ (2000) Effect of diabetes in pregnancy on offspring: follow-up research in the Pima Indians. J Matern Fetal Med 9:83-88

10. Ozanne SE, Hales CN (2002) Early programming of glucose-insulin metabolism. Trends Endocrinol Metab 13:368373

11. Berney DM, Desai M, Palmer DJ et al. (1997) The effects of maternal protein deprivation on the fetal rat pancreas: major structural changes and their recuperation. J Pathol 183:109-115

12. Aerts L, Van Assche FA (1979) Is gestational diabetes an acquired condition? J Dev Physiol 1:219-225

13. Aerts L, Van Assche FA (1992) Islet transplantation in diabetic pregnant rats normalizes glucose homeostasis in their offspring. J Dev Physiol 17:283-287

14. Goto Y, Kakizaki M, Musaki N (1976) Production of spontaneous diabetic rats by repetition of selective breeding. Tohoku J Exp Med 119:85-90

15. Galli J, Li LS, Glaser A et al. (1996) Genetic analysis of non-insulin dependent diabetes mellitus in the GK rat. Nat Genet 12:31-37

16. Gauguier D, Froguel P, Parent V et al. (1996) Chromosomal mapping of genetic loci associated with non-insulin dependent diabetes in the GK rat. Nat Genet 12:38-43

17. Gauguier D, Nelson I, Barnard C et al. (1994) Higher maternal than paternal inheritance of diabetes in GK rats. Diabetes 43:220-224 
18. Abdel-Halim SM, Guenifi A, Luthman H, Grill V, Efendic S, Ostenson CG (1994) Impact of diabetic inheritance on glucose tolerance and insulin secretion in spontaneously diabetic GK-Wistar rats. Diabetes 43:281-288

19. Gill-Randall RJ, Adams D, Ollerton RL, Alcolado JC (2004) Is human Type 2 diabetes maternally inherited? Insights from an animal model. Diabet Med:759762

20. Polychronakos C, Kukuvitis A (2002) Parental genomic imprinting in endocrinopathies. Eur J Endocrinol 147:561569
21. Plagemann A, Harder T, Franke K, Kohlhoff R (2002) Long-term impact of neonatal breast-feeding on body weight and glucose tolerance in children of diabetic mothers. Diabetes Care 25:16-22

22. Tallo CP, Vorh B, Oh W, Rubin LP, Sefier DB, Hanning RV (1995) Maternal and neonatal morbidity associated with in vitro fertilization. J Paediatr 127:794-800

23. Golombok S, Brewaeys A, Giavazzi MT, Guerra D, MacCullum F, Rust J (2002) The European study of assisted reproduction families: the transition to adolescence. Human Reprod 17:830-840 